Francisco Ledesma ${ }^{1}$

María Buti

Raquel Dominguez-

Hernández $z^{3}$

Miguel Ángel Casado ${ }^{3}$

Rafael Esteban ${ }^{2}$

\section{Is the universal population Hepatitis C virus screening a cost-effective strategy? A systematic review of the economic evidence}

\author{
${ }^{1}$ Máster en Evaluación Sanitaria y Acceso al Mercado. Universidad Carlos III, Madrid, Spain. \\ ${ }^{2}$ Hospital General Universitario Vall d'Hebron, CIBERehd, Barcelona, Spain. \\ ${ }^{3}$ Pharmacoeconomics \&t Outcomes Research Iberia (PORIB), Madrid, Spain.
}

\section{Article history}

Received: 12 March 2020; Revision Requested: 8 April 2020; Revision Received: 19 April 2020; Accepted: 12 May 2020;

Published: 8 June 2020

\begin{abstract}
Background. Efficient strategies are needed in order to achieve the objective of the WHO of eradicating Hepatitis C virus (HCV). Hepatitis $\mathrm{C}$ infection can be eliminated by a combination of direct acting antiviral (DAA). The problem is that many individuals remain undiagnosed. The objective is to conduct a systematic review of the evidence on economic evaluations that analyze the screening of HCV followed by treatment with DAAs.
\end{abstract}

Methods. Eleven databases were performed in a 2015-2018-systematic review. Inclusion criteria were economic evaluations that included incremental cost-effectiveness ratio (ICER) in terms of cost per life year gained or quality-adjusted life year.

Results. A total of 843 references were screened. Sixteen papers/posters meet the inclusion criteria. Ten of them included a general population screening. Other populations included were baby-boomer, people who inject drugs, prisoners or immigrants. Comparator was "standard of care", other high-risk populations or no-screening. Most of the studies are based on Markov model simulations and they mostly adopted a healthcare payer's perspective. ICER for general population screening plus treatment versus high-risk populations or versus routinely performed screening showed to be below the accepted willingness to pay thresholds in most studies and therefore screening plus DAAs strategy is highly cost-effective.

Conclusion. This systematic review shows that screening programmes followed by DAAs treatment is cost-effective not only for high risk population but for general population too. Because today HCV can be easily cured and its long-term

Correspondence:

Francisco Ledesma, MD

E-mail:f.ledesma@me.com consequences avoided, a universal HCV screening plus DAAs therapies should be the recommended strategy to achieve the WHO objectives for HCV eradication by 2030.

Key words: HCV, Screening, DAAs, Economic Evaluation, Cost-Effectiveness.

\section{¿Es coste-efectivo realizar el cribado poblacional para la hepatitis C? Revisión sistemática de la evidencia farmacoeconómica}

\section{RESUMEN}

Antecedentes. Para conseguir el objetivo de la Organización Mundial de la Salud (OMS) de erradicar la hepatitis C (VHC) se necesita estrategias eficientes. La infección por VHC puede ser eliminada por combinaciones de antivirales de acción directa (DAA). El problema es que muchos individuos permanecen sin diagnosticar. El objetivo es realizar una revisión sistemática de la evidencia de evaluaciones económicas que analicen el cribado del VHC (screening) seguido de tratamiento con DDA.

Método. Realizamos una revisión sistemática de once bases de datos incluyendo 2015-2018. Los criterios de inclusión fueron evaluación económica que incluyera ratio de costeefectividad incremental (ICER) en coste por año de vida ganado o año de vida ajustado por calidad.

Resultados. Extrajimos 843 referencias. Dieciséis pósters/ artículos cumplieron criterio de inclusión. Diez de ellos valoraban cribado de población general. Otras poblaciones analizadas fueron "baby-boomer", usuarios de drogas parenterales, prisiones o inmigrantes. El comparador fue "práctica clínica habitual", otras poblaciones de alto riesgo o no hacer cribado. La mayoría de los estudios utilizaron simulaciones por modelo de Markov y la perspectiva del pagador. El ICER para cribado de población general + tratamiento DDA frente a poblaciones de alto riesgo o práctica rutinaria mostraron que está por debajo del umbral de la disponibilidad a pagar en la mayoría de los estudios y conclu- 
yen que la estrategia es altamente coste-eficaz y que está por debajo de los umbrales habituales de disposición a pagar.

Conclusión. Esta revisión sistemática muestra que los programas de detección seguidos de tratamiento con DDAs son coste-efectivos no sólo para poblaciones de alto riesgo sino también para población general. Dado que hoy el VHC puede ser fácilmente curado y evitadas sus consecuencias a largo plazo, el cribado universal seguido de tratamiento con combinaciones de DDA debería ser la estrategia recomendada para alcanzar el objetivo de la OMS de erradicar el VHC para 2030.

Palabras clave: VHC, cribado, antivirales de acción directa, evaluación económica, coste-efectividad.

\section{INTRODUCTION}

World Health Organization (WHO) has recommended elimination of hepatitis $\mathrm{C}$ virus $(\mathrm{HCV})$ as a public health threat by 2030. It has been estimated that 71 million persons were living with chronic HCV in 2015 [1] and 1.75 million new HCV infections occurred in that year (global incidence rate: 23.7 per $100,000)[1,2]$. Main challenge to HCV elimination is the fact that only 20\% of the persons living with HCV had been already diagnosed, and -from them- only 7\% started treatment [1]. Population over 40 years old who once suffered certain medical interventions with low level of sterilization would remain infected and asymptomatic and represent a clear vector for HCV transmission and a risk for their future clinical decompensation. Moreover, transmission is still active in certain populations such as people who inject drugs (PWID), inmates in prisons, carriers of illegal tattoos or perinatal transmission or individuals with several sexual intercourses.

Chronic HCV infection may lead to severe and progressive systemic disease. Although chronic HCV infection is often asymptomatic, the long-term consequences are well known. WHO considered 700,000 people die each year from chronic hepatitis $C$ related-liver disease. Chronic Hepatitis $C$ infection is considered the leading cause of cirrhosis, liver cancer and liver transplant [1]. The risk of cirrhosis ranges from 15\% to $30 \%$ after 20 years of infection with HCV. Each year 1-3\% of persons with cirrhosis progress to hepatocellular carcinoma [1]. Not only liver diseases cause an extensive clinical morbidity and large economic costs, in addition, also some extrahepatic diseases, like Diabetes Mellitus, cryoglobulinemia, renal diseases, depression or chronic fatigue, also would limit the quality of life, work productivity and increases morbidity [1-10].

A major barrier to HCV eradication still results from the fact that a substantial proportion of patients with chronic HCV infection are unaware of their infection [3]. This landscape makes it crucial to consider the implementation of screening programmes.

Previously, certain economic evaluations attempted to shed light on the efficiency of implement screening programmes at diverse target population. These evaluations were conducted before direct-acting-antiviral drugs (DAAs), screening and treatment strategies when interferon/ribavirin were not a real universal treatment due to the severe tolerability issues, low efficacy and administration pathway limitations. Initially international guidelines recommended regular screening at high-risk populations like PWID or several birth cohorts (BC). A short course of DAAs have an excellent safety profile and an effectiveness up to 95\%, making possible the cure and limiting the possibility of transmission $[1,3,30]$. Therefore, screening programmes may have a broad vision and would point to general population.

In fact, WHO proposes to reduce HCV around the world and control the epidemic for 2030 [1]. In 2016, a WHO global strategy on viral hepatitis was launched, with a stated goal to eliminate hepatitis $C$ as public health threat, and bold targets for reduction in incidence and mortality by 2030: The goal of $90 \%$ reduction in new chronic infections and a $65 \%$ reduction in mortality compared to 2015 levels, highlight objectives from WHO can only be achieve setting up screening programmes around the world and covering, not only high-risk populations, but general population if possible. Population target, treatment access and cost effectiveness of the strategies are today hot topics for physicians, payers and Organizations [2].

In the current era of increasing health costs, assessment of the potential benefits of any new strategy must take into account cost-effectiveness compared to previous strategies, that is, combining long-term effects on clinical outcomes and total healthcare costs. At this scenario, pharmacoeconomic models provide the possibility to make changes in the assumptions about the design or population, they can estimate different profile populations and can be adapted at country level. Costs of the new HCV generation drugs are currently decreasing in some countries and will make it possible to treat more patients with the same budget.

The aim of this systematic review was to perform a focused review of the literature to identify relevant studies about economic evaluations for HCV screening plus treatment including DAAs strategies of the new treatment era (2015-2018).

\section{METHODS}

A systematic review was completed. Eleven databases were searched from February 2015 to April 2018 (Web of Science, MEDLINE, EMBASE, ProQuest, Premier-EBSCO, Springer Link, Google Scholar, Science Direct, Cochrane, Scopus and Open Access). Search strategies were based on the terms "HCV OR Hepatitis C" AND "screening" and "cost analysis" into the frame time of 2015-2018. Every search at the different engines were stored and filed. In addition, a manual search was carried out in abstract-book of international hepatology congresses in April 2018 to complete and update the review. Double checking by authors name were done at Medline at December 2018 in order to add final papers in case of any poster from congresses previously included were published.

Studies to be included in the systematic review need to meet the following criteria: (i) Screening plus treatment with DAAs, therapies without interferon studies. (ii) Full economic 
evaluation that contained incremental cost-effectiveness/utility ratio (ICER, ICUR) in terms of cost per life year gained or quality-adjusted life year (QALY). (iii) Written in English language. Studies were excluded if (i) They were incomplete abstracts or commentaries, (ii) Partial economic evaluations. (iii) Pure screening strategies studies focus on diagnostic methodology or epidemiology (iv) HCV treatment studies, not focus on screening, (v) Models built up based on previous interferon combinations.

For quality assessment, to evaluate the quality of the economic evaluations included we adopted the Dr Stawowczyk's methodology to evaluate the quality of the publications according to the CHEERS key points, considering "1" if they are explicit or "cero" in case this aspect does not appear properly at the paper $[5,6]$.

Data were systematically extracted. Studies were primarily grouped by year and author's alphabetical order and secondly analysed according to the population profile screened: PWID, prison population, birth cohorts (BC), general population (GP), etc. We performed a file from each study and summarised all of them in evidence tables. Data extraction was subsequently reviewed and discussed by a second member of the team.

\section{RESULTS}

During the initial search, a total of 843 potential abstracts were screened. In addition, after the EASL Conference (Paris, April 2018), twenty-five abstracts were carefully reviewed. Data duplication were removed. Some studies were discarded based on the tittle and abstract and 58 publications were fully reviewed. A total of 16 studies were ultimately identified and selected based on the inclusion/ exclusion criteria [7-23] (figure 1). Most of them were high-quality papers and abstracts according to the CHEERS's 24-item review checklist (table 1). Main pharmacoeconomic aspects were reviewed through this checklist. All of them showed measurement of effectiveness and incremental costs and outcomes, reported costs, QALYS and ICER. Eleven out of 16 showed $>90 \%$ agreement with the checklist item. Two conference abstracts initially selected were also available in full article format published before the end of 2018 and considered in both formats $[12,13,18,19]$.

The population studied was mainly general population (10 studies). At table 2 there has been summarized the study groups and the comparators [7-25]. Although population profiles are important to understand the results, also the country of origin, the local prevalence and willingness-to-pay are key

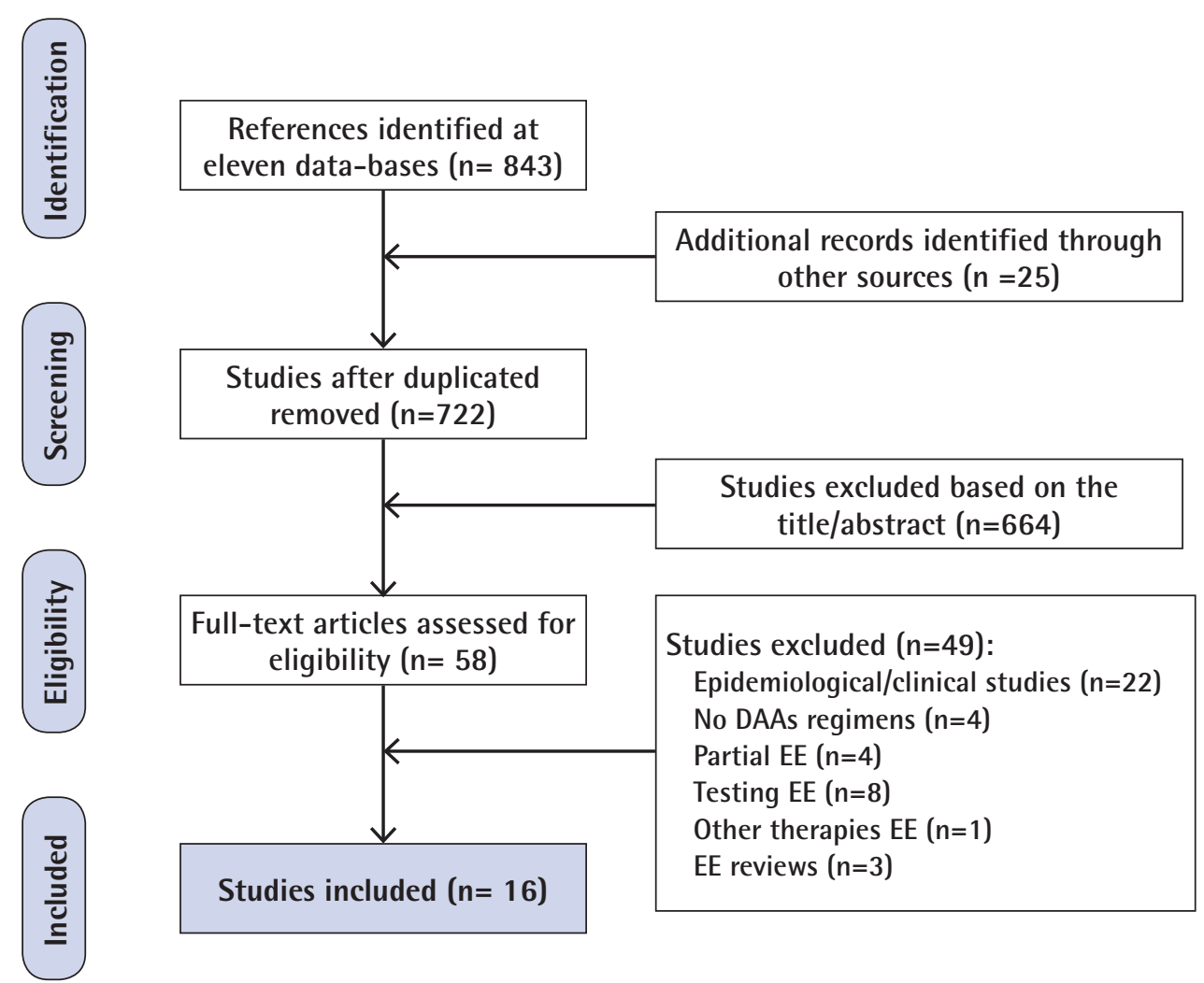

Figure $1 \quad$ Flow diagram of included studies. Preferred Reporting Items for Systematic Reviews and Meta-Analyses (PRISMA) diagram. 


\begin{tabular}{|c|c|c|c|c|c|c|c|c|c|c|c|c|c|c|c|c|}
\hline \multirow{4}{*}{ CHEERS Item no. } & \multicolumn{5}{|c|}{ USA } & \multicolumn{2}{|l|}{ France } & \multicolumn{2}{|c|}{ Korea } & \multicolumn{2}{|c|}{ Spain } & \multicolumn{2}{|c|}{ UK } & \multirow{2}{*}{$\frac{\text { Australia }}{\text { Scott }}$} & \multirow{2}{*}{$\frac{\text { Canada }}{\text { Wrong }}$} & \multirow{2}{*}{$\frac{\text { India }}{\text { Chaillor }}$} \\
\hline & $\mathrm{He}$ & Linthicum & Rattay & Younossi & Barbosa & Deuffic-Burban & Ethgen & Kim DY & Kim KA & Buti & Cuadrado & Martin & Selvapatt & & & \\
\hline & 2016 & 2016 & 2017 & 2017 & 2018 & 2016 & 2016 & 2017 & 2018 & 2018 & 2018 & 2016 & 2016 & 2017 & 2017 & 2017 \\
\hline & {$[7]$} & [8] & [9] & [10] & [11] & {$[12,13]$} & [14] & [15] & [16] & {$[18,19]$} & [20] & [21] & [22] & [23] & [24] & {$[25]$} \\
\hline 01 Title & 0 & 0 & 1 & 0 & 1 & 1 & 0 & 1 & 1 & 1 & 1 & 1 & 1 & 1 & 1 & 1 \\
\hline 02 Abstract & 1 & 1 & 1 & 1 & 1 & 1 & 1 & 1 & 0 & 1 & 0 & 1 & 1 & 1 & 1 & 0 \\
\hline 03 Background and objectives & 1 & 1 & 1 & 1 & 1 & 1 & 1 & 1 & 1 & 1 & 1 & 1 & 1 & 1 & 1 & 1 \\
\hline 04 Target population and subgroups & 1 & 1 & 1 & 1 & 1 & 1 & 1 & 1 & 1 & 1 & 1 & 1 & 1 & 1 & 1 & 1 \\
\hline 05 Setting and location & 1 & 1 & 1 & 1 & 1 & 1 & 1 & 1 & 1 & 1 & 1 & 1 & 1 & 1 & 1 & 1 \\
\hline 06 Study Perspective & 1 & 1 & 1 & 1 & 1 & 1 & 1 & 1 & 1 & 1 & 0 & 0 & 1 & 0 & 1 & 1 \\
\hline 07 Comparators & 1 & 1 & 1 & 1 & 1 & 1 & 1 & 1 & 1 & 1 & 0 & 1 & 1 & 1 & 1 & 1 \\
\hline 08 Time horizon & 1 & 1 & 0 & 1 & 1 & 1 & 1 & 1 & 1 & 1 & 0 & 1 & 1 & 1 & 1 & 1 \\
\hline 09 Discount rate & 1 & 1 & 1 & 1 & 1 & 0 & 1 & 1 & 0 & 1 & 0 & 1 & 1 & 1 & 1 & 1 \\
\hline 10 Choice of health outcomes & 1 & 1 & 1 & 1 & 1 & 1 & 1 & 1 & 1 & 1 & 0 & 1 & 1 & 1 & 1 & 1 \\
\hline 11 Measurement of effectiveness & 1 & 1 & 1 & 1 & 1 & 1 & 1 & 1 & 1 & 1 & 1 & 1 & 1 & 1 & 1 & 1 \\
\hline \multicolumn{17}{|l|}{ preference-based outcomes } \\
\hline 13 Estimating resources and costs & 1 & 1 & 1 & 1 & 0 & 1 & 1 & 1 & 1 & 1 & 1 & 1 & 1 & 1 & 1 & 1 \\
\hline 14 Currency, price date, conversion & 1 & 1 & 0 & 1 & 0 & 1 & 1 & 1 & 1 & 1 & 1 & 1 & 1 & 0 & 1 & 1 \\
\hline 15 Choice of model & 1 & 1 & 1 & 1 & 1 & 1 & 1 & 1 & 1 & 1 & 1 & 1 & 1 & 1 & 1 & 1 \\
\hline 16 Model assumptions & 1 & 1 & 1 & 1 & 1 & 1 & 1 & 1 & 1 & 1 & 1 & 1 & 1 & 1 & 1 & 1 \\
\hline 17 Analytic methods & 1 & 1 & 1 & 1 & 0 & 1 & 1 & 1 & 1 & 1 & 1 & 1 & 1 & 1 & 1 & 1 \\
\hline 18 Study parameters & 1 & 1 & 1 & 1 & 1 & 1 & 1 & 1 & 1 & 1 & 1 & 1 & 1 & 1 & 1 & 1 \\
\hline 19 Incremental costs and outcomes & 1 & 1 & 1 & 1 & 1 & 1 & 1 & 1 & 1 & 1 & 1 & 1 & 1 & 1 & 1 & 1 \\
\hline 20 Characterising uncertainty & 1 & 1 & 1 & 1 & 1 & 1 & 1 & 1 & 1 & 1 & 0 & 1 & 1 & 1 & 1 & 1 \\
\hline 21 Characterising heterogeneity & 1 & 1 & 1 & 1 & 0 & 1 & 1 & 1 & 1 & 1 & 0 & 1 & 1 & 1 & 1 & 1 \\
\hline 22 Discussion & 1 & 1 & 1 & 1 & 0 & 1 & 1 & 1 & 1 & 1 & 0 & 1 & 1 & 1 & 1 & 0 \\
\hline 23 Source of funding & 1 & 1 & 1 & 1 & 1 & 1 & 1 & 1 & 1 & 1 & 1 & 1 & 1 & 1 & 1 & 1 \\
\hline 24 Conflicts of interest & 1 & 1 & 1 & 0 & 1 & 1 & 1 & 1 & 1 & 1 & 0 & 0 & 1 & 1 & 1 & 0 \\
\hline \multirow[t]{2}{*}{ Total } & 23 & 23 & 22 & 22 & 18 & 23 & 23 & 24 & 22 & 24 & 14 & 22 & 24 & 23 & 24 & 21 \\
\hline & $98 \%$ & $98 \%$ & $92 \%$ & $92 \%$ & $75 \%$ & $98 \%$ & $98 \%$ & $100 \%$ & $92 \%$ & $100 \%$ & $58 \%$ & $92 \%$ & $100 \%$ & $98 \%$ & $100 \%$ & $88 \%$ \\
\hline
\end{tabular}

CHEERS: Consolidated Health Economic Evaluation Reporting Standards [5] and Stawowczyk's quality assessment of included studies with ISPOR CHEERS statement checklist [6]; NA: not applicable, 0 : not satisfied; 1 : satisfied.

points. At table 3 main pharmacoeconomic variables have been compilated. Discrete-time Markov model (+/- decision tree) were used in all of them to modelled HCV progression and transmission and to analyse the costs and benefits of investment in screening and treatment. Every European and Asian studies used the third-party payor perspective, most of them including a 3\% discount rate for costs and outcomes [10-13, $15,16,20,22,23]$. Some USA studies used a societal perspective including but not limited to direct costs [7-9]. Costs were reported in incremental costs per patient or in total program costs. The benefit in QALYs moved from 0.001 to 21,000 . ICER varied from AU\$47 to US\$59,589 at high-risk populations and from cost-saving to CAD $\$ 50,490$ for general population. Willingness-to-pay varies from US\$1,580 to CAD\$120,000. Studies concluded different target populations are showed to be below of accepted willingness-to-pay thresholds in the reference country (table 3). Relevantly, Younossi et al. showed general population screening to be a dominant strategy relative to birth cohort and relative to high-risk screening cohort and
ICERs moved between USD \$15,968 and \$8,660 [10]. In addition, eight of the studies described how they could prevent a great number of liver-related deaths, liver transplantations, cases of hepatocellular carcinoma, and cases of decompensated cirrhosis in the next years, according to the study horizon [7, $13,14-16,17,19-22]$. He et al. considered that, compared with no-screening, HCV screening in prisons could prevent 4,200 to 11,700 liver-related deaths, 300 to 900 liver transplantations, 3,000 to 8,600 cases of hepatocellular carcinoma, and 2,600 to 7,300 cases of decompensated cirrhosis in the next 30 years, most of the episodes outside of prison [7]. At South Korea's general population, Kim et al. estimated to avoid around 1,000 decompensated cirrhosis events and 3,000 hepatocellular carcinomas over five-year time horizon, with the largest reductions estimated in those aged $40-49$ years old at the time to diagnose and treat. A second Korean study estimated that, under a lifetime perspective, they can prevent death by 32 per 100,000 screened people $[15,16]$. In addition, Buti et al. estimated decompensating hepatic disease, transplant and death 


\section{\begin{tabular}{l|l} 
Table 2 & Summary of studies: population and comparators
\end{tabular}}

\begin{tabular}{|c|c|c|c|c|}
\hline Country & $\begin{array}{c}\text { Study, } \\
\text { Author, year [reference] }\end{array}$ & Population & Comparator & $\begin{array}{l}\text { HCV estimated } \\
\text { Prevalence }\end{array}$ \\
\hline \multirow[t]{14}{*}{ USA } & He, 2016 [7] & Prisoners: Risk-Based Screening & No-screening & $\mathrm{n} / \mathrm{a}$ \\
\hline & & All incoming inmates for up to 1 year & & \\
\hline & & All incoming inmates- 5 years & & \\
\hline & & All incoming inmates- 10 years & & \\
\hline & Linthicum, 2016 [8] & GP born before 1992 (42\% Baby boomers) & Current screening & $\mathrm{n} / \mathrm{a}$ \\
\hline & & PWID & & \\
\hline & & MSM-HIV & & \\
\hline & Rattay, 2017 [9] & GP at Primary care standard & Current practice & $1,09 \%$ \\
\hline & & GP at Primary care, ECHO project & & \\
\hline & Younossi, $2017[10]$ & GP & $B C$ & $n / a$ \\
\hline & & BC (born between 1945-1965) & HR & \\
\hline & & HR: current or past PWID & & \\
\hline & Barbosa, 2018 [11] & $\begin{array}{l}\text { PWID: scale up patients on medication assisted treatment } \\
\text { and syringe service programs }\end{array}$ & Current Practice & $\mathrm{n} / \mathrm{a}$ \\
\hline & & PWID: Scale up $+90 \%$ annual screening & & \\
\hline \multirow[t]{7}{*}{ France } & Deuffic-Burban, $2016[12,13]$ & GP $18-60$ years old & Current Screening & $\mathrm{n} / \mathrm{a}$ \\
\hline & & GP $40-80$ years old & & \\
\hline & & GP & & \\
\hline & Ethgen, 2016 [14] & BC born 1945-1965 with Low Risk of infection & Among 5 treatment strategies & $0.53 \%$ \\
\hline & & BC born 1945-1965 with Intermediate Risk of infection & (no antiviral therapy; IFN + ribavirin + protease inhibitor & \\
\hline & & BC born 1945-1965 with High Risk of infection & $\begin{array}{l}\text { for fibrosis stages F2-F4, } \\
\text { IFN-based DAAs for stages F2-F4, IFN-free DAAs for stages }\end{array}$ & \\
\hline & & & F2-F4, and IFN-free DAAs for stages F0-F4) & \\
\hline \multirow[t]{7}{*}{ South Korea } & Kim DY, 2017 [15] & GP (Age $40 \pm 49$ years) & No-Screening & $0.78 \%$ \\
\hline & & GP (Age $50 \pm 59$ years) & & \\
\hline & & GP (Age $60 \pm 69$ years) & & \\
\hline & Kim KA, 2018 [16] & GP (Age $40 \pm 65$ years) & No-Screening & \\
\hline & & GP (Age $40 \pm 49$ years) & & 0.00038 \\
\hline & & GP (Age $50 \pm 59$ years) & & 0.0061 \\
\hline & & GP (Age $60 \pm 65$ years) & & 0.0106 \\
\hline \multirow[t]{2}{*}{ Spain } & Buti, $2018[18,19]$ & GP born between 1938-1997 (20-79 years) & $\begin{array}{c}\text { General population highest anti-HCV prevalence born } \\
\text { between 1938-1967 }\end{array}$ & $0.5-1.5 \%$ \\
\hline & Cuadrado, 2018 [20] & GP at nine different age cohorts & Natural History Scenario & $1.2 \%$ \\
\hline \multirow[t]{4}{*}{ UK } & Martin, 2016 [21] & In prison: 8-week to 12-week IFN-free DAAs & In Prison: Status Quo & n/a \\
\hline & & In prison: treatment scale-up for PWID & & \\
\hline & Selvapatt, 2016 [22] & $\begin{array}{l}\text { PWID: Screening and treatment as observed within the } \\
\text { study populations (22 triple therapy, } 7 \text { DAAs) }\end{array}$ & No-Screening, No-Treatment & $\mathrm{n} / \mathrm{a}$ \\
\hline & & $\begin{array}{l}\text { PWID: Screening and treatment, assuming all patients } \\
\text { treated with hypothetical DAA therapy and SVR 95\% }\end{array}$ & & \\
\hline \multirow[t]{6}{*}{ Australia } & Scott, 2017 [23] & PWID: Scale up primary care & Current Standard of Care but with DAAs available for & Annual incidence rate \\
\hline & & PWID Scale up primary care + APRI & everyone & $11.9 \%$ \\
\hline & & PWID: Scale up primary care + APRI + annual testing of & & \\
\hline & & PWID on OST & & \\
\hline & & PWID: Scale up primary care + APRI + point of care RNA & & \\
\hline & & PWID: all heath system interventions & & \\
\hline \multirow[t]{4}{*}{ Canada } & Wong, 2017 [24] & GP & No-Screening & \\
\hline & & immigrant populations with high prevalence & & \\
\hline & & BC people aged $25-64$ years & & \\
\hline & & $B C$ aged $45-64$ years of age & & \\
\hline \multirow[t]{3}{*}{ INDIA } & Chaillon, 2017 [25] & GP at HCV prevalence $0.5 \%$ & No-Screening & $0.5 \%$ \\
\hline & & GP at HCV prevalence $1 \%$ & & $1 \%$ \\
\hline & & GP at HCV prevalence $1.5 \%$ & & $1.5 \%$ \\
\hline
\end{tabular}




\section{\begin{tabular}{l|l} 
Table 3 & Summary of cost-effectiveness results
\end{tabular}}

\begin{tabular}{|c|c|c|c|c|c|c|}
\hline $\begin{array}{l}\text { Study } \\
\text { Author, year } \\
\text { (Country) [reference] }\end{array}$ & Population & Model & Perspective & Horizon \& Discount & ICER & WTP \\
\hline $\begin{array}{l}\text { Deuffic-Burban, } 2016 \\
\text { (France) }[12,13]\end{array}$ & $\begin{array}{l}\text { GP (18-80 yr) vs } \\
\text { current-screening }\end{array}$ & $\begin{array}{l}\text { CUA, Decision tree } \\
\text { and Markov model }\end{array}$ & Societal & Lifetime, $4 \%$ & $\$ 27,600-46,300$ & $n / a$ \\
\hline $\begin{array}{l}\text { Ethgen, } 2016 \\
\text { (France) [14] }\end{array}$ & $\begin{array}{l}\text { BC born 1945-1965 } \\
\text { with different Risk of } \\
\text { infection among diverse } \\
\text { treatment approaches }\end{array}$ & $\begin{array}{c}\text { CUA/CEA, Markov } \\
\text { model }\end{array}$ & $\begin{array}{l}\text { French Health Care } \\
\text { System }\end{array}$ & 20 years, $4 \%$ & $\$ 22,986$ to $\$ 59,589$ & $\mathrm{n} / \mathrm{a}$ \\
\hline $\begin{array}{l}\text { He, } 2016 \\
\text { (USA) [7] }\end{array}$ & $\begin{array}{l}\text { Prisoners vs no- } \\
\text { screening }\end{array}$ & $\begin{array}{c}\text { CUA, microsimulation } \\
\text { model }\end{array}$ & Societal & 30 years, $3 \%$ & $\$ 19,600-29,200$ & $\$ 50,000$ \\
\hline $\begin{array}{l}\text { Linthicum, } 2016 \\
\text { (USA) [8] }\end{array}$ & $\begin{array}{l}\text { GP (born before 1992) } \\
\text { vs current-screening }\end{array}$ & CUA, Markov model & Societal & 20 years, $3 \%$ & $-\$ 6,747$ & $\mathrm{n} / \mathrm{a}$ \\
\hline $\begin{array}{l}\text { Martin, } 2106 \\
\text { (UK) [21] }\end{array}$ & Prisoners vs status quo & $\begin{array}{c}\text { CUA, Dinamic } \\
\text { transmision model }\end{array}$ & $\begin{array}{c}\text { UK National Health } \\
\text { Service }\end{array}$ & 100 years, $n / a$ & $€ 15,090-€ 6,180$ & $€ 20,000-€ 30,000$ \\
\hline $\begin{array}{l}\text { Selvapatt, } 2016 \\
\text { (UK) [22] }\end{array}$ & PWID vs no-screening & CUA, Markov model & Payer & $\begin{array}{l}100 \text { years Healthcare } \\
\text { System, } 3.5 \%\end{array}$ & $£ 1,029$ & $€ 20,000-€ 30,000$ \\
\hline $\begin{array}{l}\text { Chaillon, } 2017 \\
\text { (India) [25] }\end{array}$ & GP vs non-screening & CUA, Markov model & Heathcare Provider & Lifetime, 3\% & $\$ 1,471-2,942$ & $\$ 1,580$ \\
\hline $\begin{array}{l}\text { Kim, } 2017 \\
\text { (South Korea) [15] }\end{array}$ & $\begin{array}{l}\text { GP (Age } 40 \pm 70) \text { vs no- } \\
\text { screening }\end{array}$ & CUA, Markov model & Public Health & 5 years, $5 \%$ & $\$ 5,714-8,889$ & $\$ 27,512$ \\
\hline $\begin{array}{l}\text { Rattay, } 2017 \\
\text { (USA) [9] }\end{array}$ & GP vs current-screening & CUA, Decision tree & Societal & Lifetime, 3\% & $\$ 10,351$ & $\$ 100,000$ \\
\hline $\begin{array}{l}\text { Scott, } 2017 \\
\text { (Australia) [23] }\end{array}$ & $\begin{array}{l}\text { PWID vs current } \\
\text { standard }\end{array}$ & $\begin{array}{c}\text { CUA, Dynamic } \\
\text { compartimental model }\end{array}$ & Healthcare System & $2016-2030,0-3 \%$ & AU\$ 47 & $\mathrm{n} / \mathrm{a}$ \\
\hline $\begin{array}{l}\text { Wong, } 2017 \\
\text { (Canada) [24] }\end{array}$ & $\begin{array}{l}\text { GP (15-79 yr) vs non- } \\
\text { screening }\end{array}$ & $\begin{array}{c}\text { CUA, State transition } \\
\text { model }\end{array}$ & Third-Party Payer & Lifetime, $5 \%$ & $C \$ 31,468-50,490$ & $C \$ 50,000-120,000$ \\
\hline $\begin{array}{l}\text { Younossi, } 2017 \\
\text { (USA) [10] }\end{array}$ & $\begin{array}{c}\mathrm{GP}(>20 \mathrm{yr}) \text { vs BC } \\
\quad(1945-1967) \\
\text { and } \mathrm{GP}(>20 \mathrm{yr}) \text { vs HR }\end{array}$ & $\begin{array}{c}\text { CUA, State transition } \\
\text { model }\end{array}$ & Third-Party payer & Lifetime, 3\% & $\$ 15,968-8,660$ & $\$ 50,000$ \\
\hline $\begin{array}{l}\text { Barbosa, } 2018 \\
\text { (USA) [11] }\end{array}$ & $\begin{array}{l}\text { PWID scale up vs } \\
\text { current practice }\end{array}$ & $\begin{array}{c}\text { CUA, Two dinamic } \\
\text { compratmental models }\end{array}$ & Third-Party payer & 10 years, $3 \%$ & $\$ 6,767-11,618$ & $\$ 50,000$ \\
\hline $\begin{array}{l}\text { Buti, } 2018 \\
\text { (Spain) }[18,19]\end{array}$ & $\begin{array}{l}\text { GP }(20-79 \mathrm{yr}) \text { vs HR and } \\
\text { GP }(20-79 \mathrm{yr}) \text { vs the } \\
\text { highest prevalence }\end{array}$ & $\begin{array}{l}\text { CUA, Decision tree } \\
\text { and Markov model }\end{array}$ & National Health System & Lifetime, 3\% & $\$ 226-8,914$ & $\$ 22,000-30,000$ \\
\hline $\begin{array}{l}\text { Cuadrado, } 2018 \\
\text { (Spain) [20] }\end{array}$ & $\begin{array}{l}\text { GP (20-74 yr) vs } \\
\text { standard }\end{array}$ & $\begin{array}{c}\text { CEA Epidemiological } \\
\text { and } \\
\text { Markov model } \\
\end{array}$ & Third-Party Payer & Lifetime, n/a & $-\$ 336-3,904$ & $n / a$ \\
\hline $\begin{array}{l}\text { Kim KA, } 2018 \\
\text { (Korea) }[16]\end{array}$ & $\begin{array}{l}\text { GP (Age } 40 \pm 70) \\
\text { vs no-screening }\end{array}$ & CUA, Markov model & Healthcare System & Lifetime, 5\% & $\$ 7,218-7,787$ & $\$ 27,205$ \\
\hline
\end{tabular}

CUA: Cost-utility analysis; CEA: Cost-effectiviness analysis; n/a: not available at the paper/poster; GP: General Population; BC: Birth Cohort Population; HR: High Risk Population; PWID: people who inject drugs; ICER: incremental cost-effectiveness ratio; WTP: willingness to pay; yr: years

related would be reduced more than 50\% [17]. Nevertheless, the authors consider their model would underestimates the true clinical benefits because they did not consider improvements at the extrahepatic manifestations.

\section{LIMITATIONS}

This review has several limitations. Although an exhaustive search was performed, it is possible that not all the rele- 
vant studies were included. Only papers in English were included. The strong subject diversity at the methodology between studies and the diversity of country-characteristics make it difficult to stablish comparations and analysis. Some studies have not been yet published at paper format, and there is only poster information available; this has an impact on the CHEERS checklist punctuation. Lack of information would allow authors to use values of utilities from models that were obtained from interferon studies performed before 2015.

\section{DISCUSSION}

WHO proposes to eradicate viral hepatitis as a public health threat by 2030. In order to fulfil this objective, HCV screening programmes seems to be a leverage point. The evidence to propose a regular screening to a wide population is low, and recommendations are sometimes conditional. If eradication of chronic HCV infection as a public health threat requires diagnosing 90\% of those infected and treating $80 \%$ of those diagnosed, new campaigns to introduce and reinforce testing and treatment should be implemented in order to address this important gap [1].

Barriers imposed by previous treatments (i.e., sub-optimal efficacy, inadequate tolerability, longer duration of treatment) are no longer applicable. Screening programmes would make possible the strategies to "one-time-screening" and to "test and treat". Thanks to the DAAs regimens, treatment after screening can be simpler, shorter, safer and better tolerated, so rejection from HCV infected patients would be expected to be very low, while cure rate is expected in almost every patient who completes treatment.

Good quality screening systematic reviews were previously published in the past [26-31], analysed interferon-based therapies with a suboptimal tolerability profile and lower efficacy. The scenario have changed with the current DAAs treatments that allow to increase the number and characteristics of HCV infected subjects that can receive therapy. According to that, there are needed of cost-effectiveness data including general population evaluation arms. For this reason, we performed the current systematic review, traying to put in perspective the screening evidences at the current DAAs treatment era. Cost-effectiveness ratio (ICER) is highly favourable at most of the comparations, being screening plus DDAs treatment a cost-effective strategy not only for prisoners, inmates who left the prison and PWID, but also cost-effective for selected birth cohort populations and general population. Most of the model results are considered to be below the willingness-to-pay threshold [7-25]. Studies considered some populations at different regions. This broad representation means that the topic interests to diverse economic and health system settings. Although the implementation of the strategy implies an increase of current budget because cost increases for the first years, it seems that screening campaigns would break even in less than ten years [8]. From a clinical perspective, long term clinical complications particularly hepatocellular carcinoma will reduce but not abolish in the next years and savings have to be analysed in future studies that also include the impact of extra-hepatic disease, tiredness, mood disturbances (like anxiety, and depression), health related quality of life (HROL) and work productivity impact.

The cost of the DAA drugs has been a "hot" topic and it impacted to the current cost-effectiveness studies. Fortunately, prices have dropped around the world and it is estimated that they will continue to decrease even further. Most models include a sensitivity analysis that incorporates a 20\% decrease in DAA cost. Reducing costs at the sensitivity analysis means conclusions of the models are even more cost-effective. This scenario would be the real access situation for the next years, providing the possibility to screen and treat more patients with the same budget. Other key factors that showed more impact at the analysed studies were the local prevalence, the age at diagnosis, the time between test and treatment and the fibrosis level. These factors are aligned with the current WHO recommendations that suggest not delay the HCV treatment after positive screening.

The access to HCV testing and therapy is still suboptimal in many countries due to the disease is asymptomatic for many years. At this point, the one-step testing shows a great initiative that support an easier and shorter screening methodology, losing less patients in the process. In addition, several studies promote strategies to involve more in deep to primary care services at the HCV management $[9,23]$, reducing the number of patients lost and promoting early treatment under a "test and treat initiative". It is crucial that at country level this pathway and sensitivity would be designed and implemented under this new scenario and possibilities. Several success examples, like the Scottish initiative (2006), Germany (2013), Italy and Spain (2015) have increased the fight against HCV infection [32]. A recent example would be the Spanish National Plan for HCV which achieve treatment to more than 117,500 patients in around three years, with a shared effort between payors, pharmaceutical companies and physicians [33]. They are now focused on increasing one-time diagnostic process among asymptomatic individuals in order to reduce the number of silent infections, increasing new diagnosis while avoiding losing patients in the process. These local collaborative initiatives may constitute the framework to design a strategy for the future. This strategy would need to be supported by local epidemiological and cost-effectiveness studies taking into account their own resources.

Like other infectious diseases, preventive medicine allows society to get ahead the disease, preventing its evolution and avoiding suffering, death and costs. From the moment that effective and well tolerated therapies are available, HCV screening and treatment cost-effectiveness requires to be evaluated as a successful strategy at the general population level. Although more evidence is needed at each country level, the current data suggest that screening recommendations for the general population should be included on international guidelines as a major public health strategy. 


\section{CONCLUSION}

An HCV strategy of "test and treat" is cost-effective for screening of HCV high risk population and also for the general population. Because currently HCV can be easily cured, and the majority of long-term consequences can be avoided, a universal HCV screening plus DAAs treatment should be the recommended strategy to achieve the WHO objectives of HCV eradication by 2030.

\section{ACKNOWLEDGEMENTS}

The authors thank Pilar Alonso, from the Library of The Carlos III University (Madrid) who supported us on the search process and document management. Ángeles Flores provided expert imput on the database seaching process, identifying the best approach for the final search at each database. Guy de la Rosa provided scientific review feedback.

\section{CONFLICTS OF INTEREST}

Maria Buti is an advisor for Gilead Sciences, Abbvie and MSD. Rafael Esteban is an advisor for Gilead Sciences, Abbvie and MSD. Francisco Ledesma worked at Janssen until more than two years before this study was started. Raquel Domínguez-Hernández and Miguel Ángel Casado are employees of Pharmacoeconomics \&t Outcomes Research Iberia, a consultancy firm specialising in the economic evaluation of healthcare interventions, which has received unconditional funding from Gilead Sciences.

\section{FUNDING}

None to declare

\section{REFERENCES}

1. WHO. Guidelines for the screening, care and treatment of persons with chronic hepatitis C infection. Updated version, July 18, 2018. http://www.who.int/news-room/fact-sheets/detail/hepatitis-c. Accessed on 18 July 2018.

2. WHO Global Health Sector Strategy on Viral Hepatitis 2016-2021: Towards Ending Viral Hepatitis. 2016. http://www.who.int/ hepatitis/strategy2016-2021/ghss-hep/en. Version June 2016. Accessed on 22 August 2018.

3. European Association for the Study of the Liver. EASL Recommendations on Treatment of Hepatitis C 2018. J Hepatol 2018; 69(2): 461-511. PMID: 29650333.

4. AASLD. HCV Guidance: Recommendations for Testing, Managing, and Treating Hepatitis C. 2018 (24 May). https://www.hcvguidelines.org. Accessed on 28 August 2018.

5. Husereau D, Drummond M, Petrou S, Carswell C, Moher D, Greenberg D, et al.; CHEERS Task Force. Consolidated Health Economic Evaluation Reporting Standards (CHEERS) statement. Value Health 2013;16(2):e1-5. PMID: 23538200.
6. Stawowczyk E, Kawalec P. A Systematic Review of the Cost-Effectiveness of Biologics for Ulcerative Colitis. PharmacoEcon Open 2018;36:419-434. PMID: 29260508.

7. He T, Li K, Roberts MS, Spaulding AC, Ayer T, Grefenstette JJ, et al. Prevention of hepatitis $\mathrm{C}$ by screening and treatment in US Prisons. Ann Intern Med 2016; 164: 84-92. PMID: 26595252.

8. Linthicum MT, Gonzalez YS, Mulligan K, Moreno GA, Dreyfus D, Juday $T$, et al. Value of expanding HCV screening and treatment policies in the United States. Am J Manag Care 2016; 22:SP227-35. PMID:27266953.

9. Rattay $T$, Dumont IP, Heinzow HS, Hutton DW. Cost-Effectiveness of Access Expansion to Treatment of Hepatitis C Virus Infection Through Primary Care Providers. Gastroenterology 2017; 153(6):1531-1543. PMID:29074450.

10. Younossi ZM, Blissett D, Blissett R, Henry L, Younossi Y, Beckerman $R$, et al. In an era of highly effective treatment, hepatitis $C$ screening of the United States general population should be considered. Liver int 2018;38:258-265. PMID:28719013.

11. Barbosa C, Fraser $H$, Hoerger $T$, Leib $A$, Havens $L R$, Young $A$, et al. Cost-effectiveness of scaling up HCV prevention, testing and treatment among people who inject drugs in the US. EASL 2018, Paris. J Hepatol 2018; 68. Poster THU-131.

12. Deuffic-Burban $S$, Huneau A, Verleene A, Brouard C, Pillonel J, Le Strat $Y$, et al. Cost-Effectiveness of screening strategy of hepatitis C in France: It is time to change Recommendations. AASLD 2016 Hepatology 2016; 64 (1 Supplement 1): 375A-376a.

13. Deuffic-Burban $S$, Huneau A, Verleene A, Brouard C, Pillonel J, Le Strat $Y$, et al. Assessing the cost-effectiveness of hepatitis $C$ screening strategies in France. J Hepatol 2018;69(4):785-792. PMID:30227916.

14. Ethgen 0 , Sanchez Gonzalez $Y$, Jeanblanc G, Duguet A, Misurski D, Juday T. Public health impact of comprehensive hepatitis $C$ screening and treatment in the French baby-boomer population. J Med Econ 2017;20(2):162-170. PMID:27590836.

15. Kim DY, Han K-Y, Jun B, Kim TH, P S, Ward T, Webster S, et al. Estimating the cost-Effectiveness of one-Tine Screening and Treatment for Hepatitis C in Korea. Plos One 2017; 12(1):e0167770. PMID:28060834.

16. Kim KA, Chung W, Choi HY, Jang ES, Ki M, Jeong SH. Cost-effectiveness of screening for hepatitis $\mathrm{C}$ in Korean General Population. EASL 2018, Paris. J Hepatol 2018; 68. Poster THU-433.

17. Kim KA, Chung W, Choi HY, Ki M, Jang ES, Jeong SH. Cost-effectiveness and health-related outcomes of screening for hepatitis $C$ in Korean population. Liver Int 2019; 39: 60-69. PMID:29998565.

18. Buti M, Domínguez-Hernández R, Casado MA, Sabater E, Esteban R. Screening and treatment of hepatitis $C$ in adults of general population in Spain is cost-effective. EASL 2018, Paris. J Hepatol 2018; 68. Poster THU-140.

19. Buti M, Domínguez-Hernández R, Casado MA, Sabater E, Esteban R. Healthcare value of implementing hepatitis $\mathrm{C}$ screening in the adult general population in Spain. PLoS One 2018;13(11):e0208036. PMID:30485377.

20. Cuadrado A, Perello C, Llerena S, Gomez M, Escudero MD, Rodriguez $L$, et al. Design and cost effectiveness of a hepatitis $C$ virus elimi- 
nation strategy based on an updated epidemiological study (ETHON cohort). EASL 2018, Paris. J Hepatol 2018; 68. Poster THU-087.

21. Martin NK, Vickerman P, Brew IF, Williamson J, Miners A, Irving $W L$, et al. Is increased hepatitis $C$ virus case-finding combined with current or 8-week to 12-week direct-acting antiviral therapy cost-effective in UK prisons? A prevention benefit analysis. Hepatology 2016; 63(6):1796-808. PMID:26864802.

22. Selvapatt N, Ward T, Harrison L, Lombardini, J, Thursz M, McEwan P, et al. The cost impact of outreach testing and treatment for hepatitis C in an urban Drug Treatment Unit. Liver Int 2017; 37: 345-353. PMID:27566283.

23. Scott N, Doyle JS, Wade A, Howell J, Pedrana A, Thompson A, et al. Reaching hepatitis $C$ virus elimination targets requires health system interventions to enhance the care cascade. Int J Drug Policy 2017;47:107-116. PMID:28797497.

24. Wong W, Erman A, Feld J, Krahn M. Model-based projection of health and economic effects of screening for hepatitis $\mathrm{C}$ in Canada. CMAJ open 2017; E662-E672. PMID:28851700.

25. Chaillon $A$, Mehta SR, Hoenigl M, Vickerman P, Hickman $M$, Skaathun B, et al. The Cost-effectiveness of General Population HCV Screening in India. 68th AASLD, Washington 2017. Hepatology 2017; 66 (765): 410a.

26. Camera A, lanuale C, Boccia S. Cost-effectiveness of HCV screening: a systematic review of the literature from 2007 to 2012. Epidemiol Biostat Public Health 2013; 10 (3): e8767-e8768.

27. Coretti S, Romano F, Orlando V, Codella P, Prete S, Di Brino E, et al. Economic evaluation of screening programs for hepatitis $C$ virus infection: evidence from literature. Risk Manag Healthc Policy 2015 21;8:45-54. PMID:25960681.

28. Coward S, Leggett L, Kaplan GG, Clement F. Cost effectiveness of screening for hepatitis C virus: a systematic review of economic evaluations BMJ Open 2016;6(9):e011821. PMID:27601496.

29. Geue C, Wu O, Xin Y, Heggie R, Hutchinson S, Martin NK, et al. Cost-Effectiveness of HBV and HCV Screening Strategies - A Systematic Review of Existing Modelling Techniques. PLoS One 2015 10(12):e0145022. PMID:26689908.

30. Morgan JR, Servidone M, Easterbrook P, Linas BP. Economic evaluation of HCV testing approaches in low and middle income Countries. BMC Infectious Diseases 2017 17(Suppl 1):697: 117-127. PMID:29143681.

31. CADTH Heath Technology Assessment Screening for Hepatitis C Virus: A Systematic Review. 2017 March. https://www.ncbi.nlm.nih. gov/pubmedhealth/PMH0098272/ Accessed on 28 August 2018.

32. Papatheodoridis GV, Hatzakis A, Cholongitas E, Baptista-Leite R, Baskozos I, Chhatwal J, et al. Hepatitis C: The beginning of the end-key elements for successful European and national strategies to eliminate HCV in Europe. J Viral Hepat 2018; 25 Suppl 1:6-17. PMID:29508946.

33. Buti M, Calleja JL, Garcia-Samaniego J, Serra MÁ, Crespo J, Romero $M_{1}$ et al. Elimination of hepatitis $C$ in Spain: Adaptation of a mathematical model based on the public health strategic plan for addressinghepatitis C in the National Health System. Med Clin (Barc) 2017; 148(6):277-282. PMID:28159349. 